transistor (SSET) displacement detector ${ }^{9}$. The SSET can similarly in principle resolve micromechanical motion approaching the standard quantum limit by taking advantage of its sensitivity to charge fluctuations that are orders of magnitude smaller than a single electron charge.

Assuming that we will soon have a near-quantum-limited SQUID or other type of displacement detector, what can it be used for? Ultimately, the goal will be to verify the hitherto untested quantum nature of micrometre-scale mechanical resonators. However, in the intermediate term, it will be very interesting to probe the low-temperature (corresponding to temperatures of just a few tens of millikelvin and less) and small-amplitudedisplacement damping mechanisms of the megahertz radiofrequency mechanical resonators. Recent experiments ${ }^{10}$ with much larger, kilohertz frequency resonators suggest that tunnelling two-level system (TLS) defects have an important role in mechanical damping, although there is also significant disagreement with standard theories of damping due to TLSs. With quantum-limited displacement sensitivities, it should be possible to shed considerable light on TLS and other damping mechanisms, which will in turn eventually help us to understand how classical dynamics arises from quantum dynamics for actual mechanical systems.

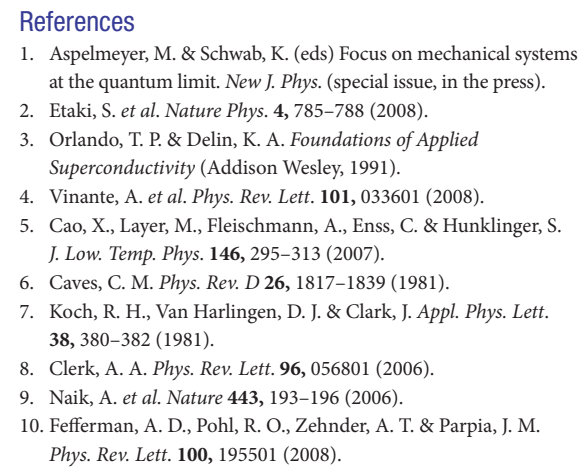

\title{
MAGNETOELECTRONICS
}

\section{An attractive way to change direction}

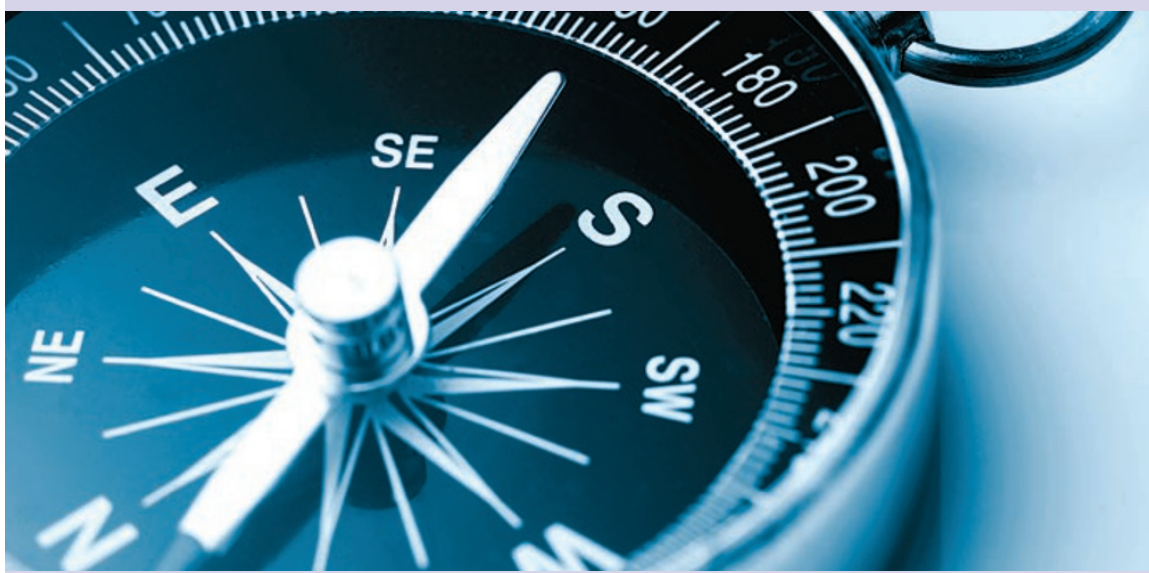

Storage media are typically made from magnetic materials in which information is encoded through the orientation of the magnetization vector. The magnetization is usually manipulated by current-generated magnetic fields but, for integration into conventional electronics, it is desirable to be able to change the direction of the magnetization using electric fields alone. This kind of control has now been demonstrated by Daichi Chiba and colleagues in a metal-insulatorsemiconductor system (Nature 455, 515-518; 2008).

The device is based on a thin film of the magnetic semiconductor gallium manganese arsenide (GaMnAs). Magnetic semiconductors combine interesting ferromagnetic and semiconducting properties, and GaMnAs is a wellstudied example.

When manganese atoms are introduced into the GaAs host material, they replace individual gallium atoms; but as manganese has one fewer valence electron, this results in positively charged 'holes', which are free to move about in the material and contribute to its conductive properties. Importantly, the holes provide a mechanism for long-range ferromagnetic interactions between the magnetic manganese ions, which behave like localized spins. The holemediated spin-spin interaction is sensitive to the charge-carrier concentration and thus the material's ferromagnetic properties can be controlled using an electric field - as has been demonstrated, for example, for the ferromagnetic transition temperature.

Chiba et al. have gone further and demonstrate electric-field control of the orientation of the magnetization in the absence of any external magnetic field. Their system is simple: a thin film of ferromagnetic GaMnAs separated by a thin insulator from a metal gate electrode, through which an electric field can be applied. Because of the compressive strain in the film, it is energetically more favourable for the localized spins of the manganese atoms to be oriented in the plane of the film. However, the direction of magnetization in the plane is governed by less intuitive mechanisms.

Owing to the crystallographic and electronic structure of the material, there are several so-called magnetic easy axes - the magnetic anisotropy energy is at a minimum when the magnetization is parallel to one of these axes - and the direction of the magnetization is determined by competition between them. Earlier theoretical and experimental work has shown that the direction of the magnetic easy axes themselves can be changed by tuning hole concentration, strain or temperature.

Chiba et al. tweaked the hole concentration in their system using the voltage applied to the metal gate electrode; through careful transport measurements, they determined the magnetic anisotropy constants describing the 'strength' of the magnetic easy axes, and, more importantly, proved that the resulting orientation of the magnetization can be sensitively tuned by the applied electric field.

This approach is certainly attractive in its simplicity, and complements other attempts to control magnetism using electric fields, such as through magnetostriction, spin currents or multiferroic coupling. So far, however, the tuning of the magnetization angle has been achieved only in a restricted range, and at cryogenic temperatures. The challenge remains to achieve efficient electric-field switching of magnetization at room temperature.

Dan Csontos 